This document is the accepted manuscript version of the following article:

Rota, L., Case11e, M., Bründermann, E., Funkner, S., Gerth, C., Kehrer, B., ... Weber, M. (2019). KALYPSO: Linear array detector for high-repetition rate and real-time beam diagnostics. Nuclear Instruments and Methods in Physics Research, Section A: Accelerators, spectrometers, Detectors and Associated Equipment, 936, 10-13. https://doi.org/10.1016/j.nima.2018.10.093

This manuscript version is made available under the CC-BY-NC-ND 4.0 1icense http://creativecommons . org/1icenses/by-nc-nd/4.0/

\title{
KALYPSO: Linear array detector for high-repetition rate and real-time beam diagnostics
}

\author{
L. Rota ${ }^{\text {a, }{ }^{* 1}, \text { M. Caselle }}{ }^{\text {a }}$, E. Bründermann ${ }^{\text {a }}$, S. Funkner ${ }^{\mathrm{a}}$, Ch. Gerth ${ }^{\mathrm{b}}$, B. Kehrer ${ }^{\mathrm{a}}$, \\ A. Mielczarek ${ }^{\mathrm{d}}$, D. Makowski ${ }^{\mathrm{d}}$, A. Mozzanica ${ }^{\mathrm{c}}$, A.-S. Müller ${ }^{\mathrm{a}}$, M.J. Nasse ${ }^{\mathrm{a}}$, G. Niehues ${ }^{\mathrm{a}}$, \\ M. Patil ${ }^{\text {a }}$, B. Schmitt ${ }^{\text {c }}$, P. Schönfeldt ${ }^{\mathrm{a}, 2}$, B. Steffen ${ }^{\mathrm{b}}$, M. Weber ${ }^{\mathrm{a}}$ \\ ${ }^{\text {a }}$ Karlsruhe Institute of Technology - KIT, Karlsruhe, Germany \\ ${ }^{\mathrm{b}}$ Deutsches Elektronen-Synchrotron - DESY, Hamburg, Germany \\ c Paul Scherrer Institute - PSI, Villigen, Switzerland \\ ${ }^{\mathrm{d}}$ University of Łódź, Ł,ódź, Poland
}

\section{A R T I C L E I N F O}

Keywords:

Line scan detector

Micro-strip detector

Ultra-fast imaging

\begin{abstract}
A B S T R A C T
Synchrotrons and modern FEL light sources operate with bunch repetition rates in the MHz range. The profile of the electron beam inside the accelerator can be characterized with indirect experimental techniques where linear array detectors are employed to measure the emitted synchrotron radiation or the spectrum of a near-IR laser. To improve the performance of modern beam diagnostics we have developed KALYPSO, a detector system operating with a continuous frame rate of up to $2.7 \mathrm{MHz}$. To facilitate the integration in different experiments, a modular architecture has been adopted. Different semiconductor micro-strip sensors can be connected to front-end ASICs to optimize the quantum efficiency at different photon energies, ranging from visible light up to near-IR. The front-end electronics are integrated within an heterogeneous DAQ consisting of FPGAs and GPUs, which allows scientists to implement real-time data processing algorithms. The current version of the detector is in operation at the KARA synchrotron light source and at the European XFEL. In this contribution we present the detector architecture, the performance results and the on-going technical developments.
\end{abstract}

Contents

1. Introduction

10

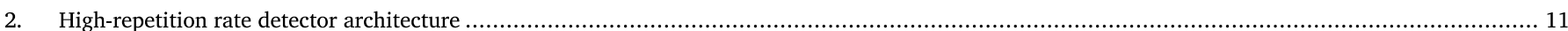

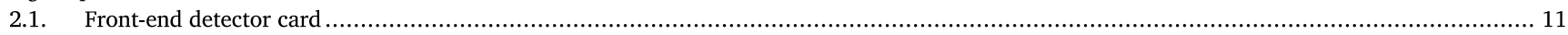

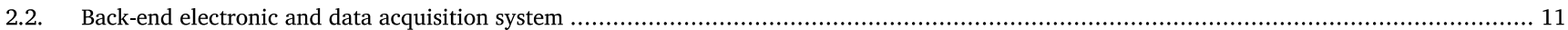

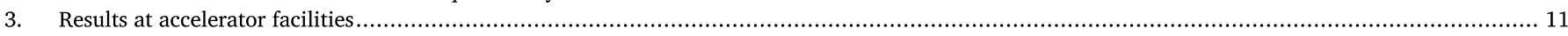

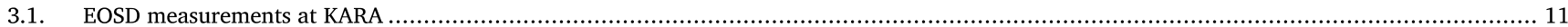

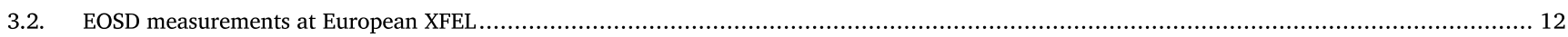

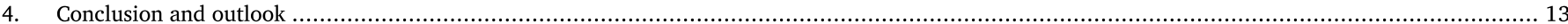

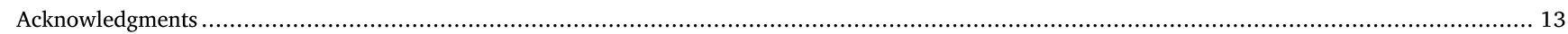

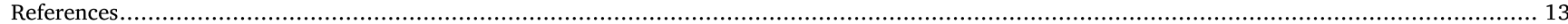

\section{Introduction}

The term "beam diagnostics" identifies systems which measure the proprieties of a beam of charged particles inside an accelerator. An example of such techniques is Electro-Optical Spectral Decoding (EOSD) [1], which allows scientists to probe the structure of electron bunches in a non-destructive way and with high temporal resolution. In short, the EOSD technique exploits an electro-optical effect to encode the longitudinal profile of an electron bunch on the frequency spectrum of a laser pulse. The spectrum of the laser pulse is then analyzed in a spectrometer equipped with a line scan detector. A temporal resolution down to $200 \mathrm{fs}$ rms has been achieved [2].

\footnotetext{
* Corresponding author.

E-mail addresses: lorenzo.rota@kit.edu (L. Rota), michele.caselle@kit.edu (M. Caselle).

1 Now at SLAC National Accelerator Laboratory, Menlo Park, CA, United States.

2 Now at DLR-VE, Oldenburg, Germany.
} 
In order to enable single-shot measurements on consecutive bunches, the frame rate of the line scan detector mounted in the spectrometer has to match the repetition rate of the accelerator. Such repetition rates span from a few $\mathrm{MHz}$ at FEL facilities (e.g., $4.5 \mathrm{MHz}$ at the European X-ray Free Electron Laser) to several hundreds of $\mathrm{MHz}$ at synchrotron light sources (e.g., $500 \mathrm{MHz}$ at the Karlsruhe Research Accelerator (KARA) with a multi-bunch filling scheme, or $2.7 \mathrm{MHz}$ with a single-bunch), posing several technological challenges for the development of a suitable detector and data acquisition system.

The solution adopted by high-resolution imagers intended for use at the Eu-XFEL and operating at $\mathrm{MHz}$ rates [3] is to add an analog or digital memory in the front-end electronics. In this way, only a limited number of images is acquired (e.g., up to 352 images with the adaptive gain integrating pixel detector [3]) at a frame rate of $4.5 \mathrm{MHz}$, which are then stored in the local memory before being read-out at a slower speed by the data acquisition (DAQ) system. While this is a solution adopted for experiments running with a bunch train scheme, other facilities and experiments would greatly benefit from the possibility to acquire a continuous number of samples at $\mathrm{MHz}$ repetition rates, as is the case with synchrotron light sources.

Moreover, the presence of memories in the signal path introduces a certain latency in the readout phase. This additional latency is typically not acceptable in beam diagnostics applications, where data produced by the detector is employed to apply a fast-feedback on the accelerator. Minimizing the total latency of the system is of critical importance towards the goal of implementing fast-feedback control loops operating at $\mathrm{MHz}$ rates and based on single-shot spectral measurements.

In this contribution we present KALYPSO (KArlsruhe Linear arraY detector for MHz-rePetition rate SpectrOscopy), a line scan detector for visible and near-infrared (IR) light operating at a frame-rate of $2.7 \mathrm{MHz}$. The main feature of KALYPSO with respect to other detector systems is the streaming-mode operation at $\mathrm{MHz}$ frame rates, during which data are continuously acquired and processed with minimal latency. The detector has been developed in collaboration between the Łódź University of Technology (TUL), Paul Scherrer Institute (PSI), Deutsches Elektronen-Synchrotron (DESY) and Karlsruhe Institute of Technology (KIT), with the goal to improve the temporal resolution of the EOSD experimental setups installed at KARA and Eu-XFEL.

Section 2 describes the detector architecture and its integration with a DAQ framework for real-time data processing based on FPGAs and GPUs. Two examples of applications of KALYPSO as beam diagnostic tool are given in Section 3, where preliminary measurements taken at KARA and at the Eu-XFEL are reported. Finally, we give an outlook on ongoing technical developments in Section 4.

\section{High-repetition rate detector architecture}

The KALYPSO detector system has been designed following a modular approach to enable the integration with different data acquisition systems at different facilities. It consists of a mezzanine card, which hosts the sensor and the front-end electronics, and a Field Programmable Gate Array (FPGA) data acquisition and processing board, which connects the detector to the DAQ system. A picture of the KALYPSO frontend card is shown in Fig. 1.

\subsection{Front-end detector card}

A hybrid detector architecture has been chosen to meet the requirements of different experiments, which employ light sources with different wavelengths. Two different types of semiconductor sensors can be equipped on the printed circuit board (PCB). The first type is a $\mathrm{Si}$ microstrip sensor which has been designed at PSI and is suitable for wavelengths up to $1050 \mathrm{~nm}$. For experiments working with wavelengths between $1050 \mathrm{~nm}$ and $1.7 \mu \mathrm{m}$, KALYPSO is equipped with a commercial InGaAs sensor produced by Xenics. The channel pitch of both sensors is
$50 \mu \mathrm{m}$, matching the channel pitch of the readout ASIC, for a total of 256 strips.

The current version of KALYPSO mounts a modified version of the GOTTHARD chip [4] as front-end ASIC. This version has been developed to verify the feasibility of the detector system and the performance of the ASIC with InGaAs sensors, which exhibit a strip capacitance of up to $8 \mathrm{pF}$.

The ASIC features 128 channels, each one consisting of a chargesensitive amplifier (CSA) and a channel buffer. A 16:1 analog multiplexer connects 16 channels to a differential output driver which interface the external ADC. With respect to the original GOTTHARD chip, some features such as the Correlated-Double-Sampling (CDS) stage and automatic gain switching mechanism have been omitted, and the power budget of the chip has been re-allocated to achieve a higher analog bandwidth. In this way the maximum frame-rate achievable is $2.7 \mathrm{MHz}$, with an improvement of almost a factor of 3 with respect to the original GOTTHARD design. However, the higher bandwidth and lack of a CDS noise shaper result in a higher noise contribution. The Equivalent Noise Charge (ENC) of the readout ASIC when connected to a detector capacitance of $1.3 \mathrm{pF}$ has been estimated to be around 600 electrons with post-layout simulations.

The sensors are connected to the front-end ASIC with Au ball-towedge wire bonds with a diameter of $20 \mu \mathrm{m}$, while the connection between the ASIC and the PCB is realized with standard Al wedge-towedge wire bonds.

The analog outputs are digitized by an AD9249 ADC with a sampling rate of $65 \mathrm{MS} / \mathrm{s}$ and a resolution of 14 bits. In addition, the PCB is equipped with digital-to-analog converters (DAC) to provide bias references to the front-end ASIC and with a clock conditioner which generate a low-jitter clock synchronous to the input clock, which is generated by the timing system of the accelerator.

\subsection{Back-end electronic and data acquisition system}

The mezzanine card is connected through a VITA 57.1 FMC connector to an "High-Flex" board [5], a custom FPGA-based readout card developed at KIT. The card mounts a Xilinx Virtex-7 xc7vx690t device and features two fully-populated FMC connectors, a 4 GB DDR3 memory and two PCI Express Gen3 x8 interfaces.

Digitized data coming from the ADC are acquired by the FPGA, where different digital signal processing stages can be implemented according to the specific application. The DDR3 memory acts as a local memory buffer, which can be disabled if low-latency data transmission is required.

KALYPSO is integrated in an heterogeneous DAQ system consisting of FPGAs and GPUs directly connected through PCI Express interface [6]. A custom Direct Memory Access (DMA) engine allows direct FPGA-GPU communication with a maximum throughput of $7.2 \mathrm{~GB} / \mathrm{s}$, exceeding the data rate of $1.4 \mathrm{~GB} / \mathrm{s}$ produced by one KALYPSO system at the maximum frame rate ( 14 bits $\times 256$ pixels at $2.7 \mathrm{Mfps}$ ) [7].

Low-latency data analysis on GPUs was demonstrated in [8], with a latency ranging from a few to several tens of microseconds, depending on the particular algorithm implemented. With respect to traditional approaches based on FPGAs, the use of GPUs allows users without deep expertise in digital logic design to deploy a specific experiment.

\section{Results at accelerator facilities}

\subsection{EOSD measurements at KARA}

KALYPSO has been installed at the EOSD experimental setup at KARA, as shown in Fig. 2. A Yb-doped fiber laser generates pulses with a repetition rate of $2.7 \mathrm{MHz}$ (corresponding to the bunch revolution frequency $f_{\text {rev }}$ ) and with a wavelength of $1050 \mathrm{~nm}$. The laser pulses are sent to a GaP crystal in the Ultra-High-Vacuum (UHV) section of the storage ring and then back to the EO hutch for detection and analysis. 


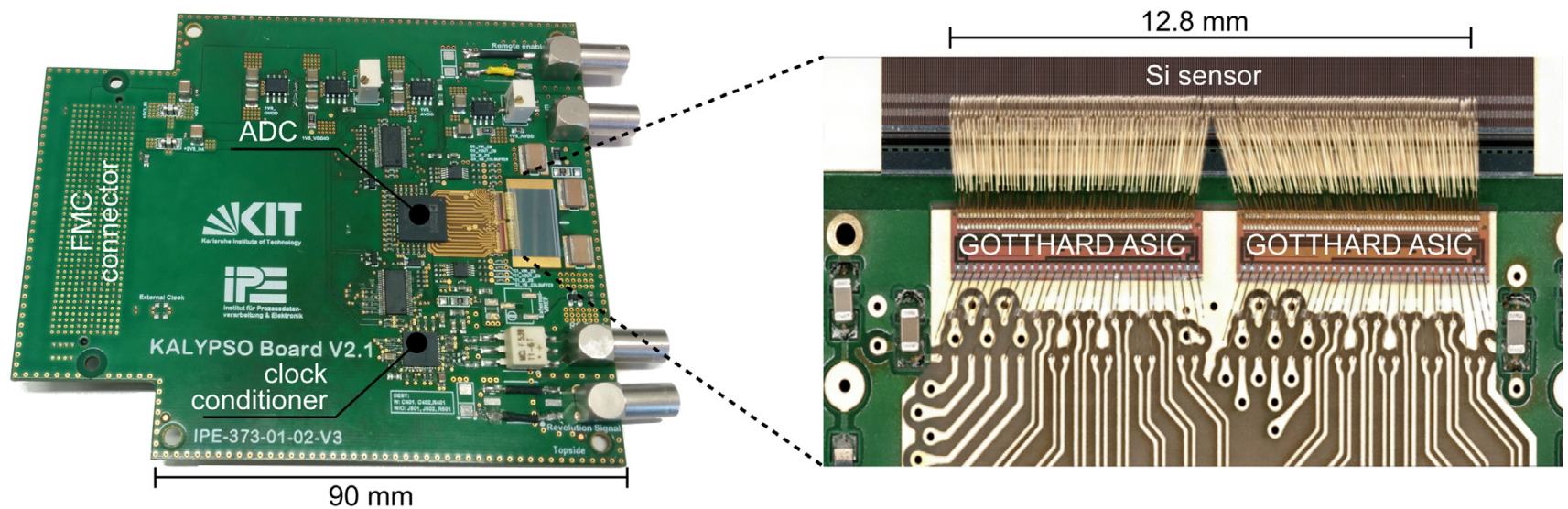

Fig. 1. Picture of the KALYPSO front-end card (left) and the high-density wire-bonding interconnections between the Si sensor, the two GOTTHARD ASICs and the high-density interconnection board.

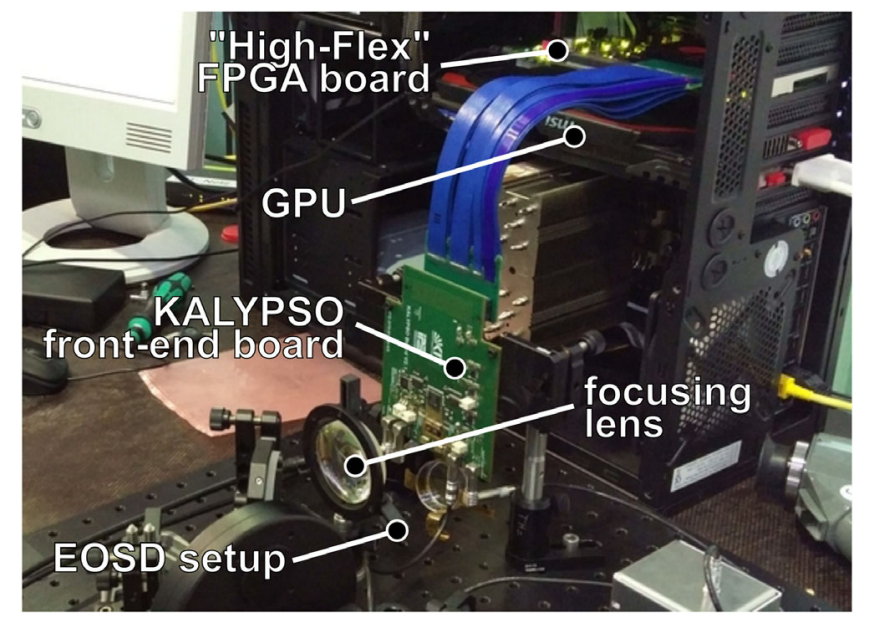

Fig. 2. Picture of the KALYPSO detectors installed at the EOSD experimental setup at KARA.

The underlying principle of EOSD is based on the Pockel effect: the $\mathrm{GaP}$ crystal exhibits birefringence in presence of a strong electric field. When both the Coulomb field of the electron bunch and the laser pulse propagate in the crystal at the same time, the spectrum of the chirped laser is modulated. A detailed description of the principle of operation can be found in [9]. The laser is then sent back to the experimental hutch and analyzed in a spectrometer equipped with the KALYPSO detector to retrieve the temporal information on the longitudinal bunch profile.
Fig. 3 shows a measurement of the longitudinal bunch profile obtained with KALYPSO equipped with a Si sensor and operating at the maximum frame-rate of $2.7 \mathrm{MHz}$. The streaming capability of KALYPSO enables the acquisition of up to $10^{7}$ frames, allowing scientists to analyze the beam dynamics over a large range of frequencies. Further details on the setup and on the data analysis have been published in [10].

\subsection{EOSD measurements at European XFEL}

A compact EOSD setup [11] has been installed in the injector of the European XFEL. The setup comprises an identical $\mathrm{Yb}$-doped fiber laser, as described above for the experiment at KARA, and a KALYPSO detector system for the spectral measurement of the modulated laser pulses. The back end electronics is different than the one described in the previous sections, since it has to connect to the MicroTCA.4 standard in use at the E-XFEL [12]. The laser has been synchronized to the accelerator frequency of $1.3 \mathrm{GHz}[13,14]$. A thickness of $5 \mathrm{~mm}$ was chosen for the GaP crystal to match the expected electron bunch lengths in the injector. Fig. 4 depicts the measured modulation of the laser pulses recorded with a frame rate of $1.13 \mathrm{MHz}$. The last 26 frames, for which no electron bunches were present, are only shown for demonstration. The modulation depth of the 500 consecutive electron bunches shown in color code amount $-5 \%$ (blue) to $+10 \%$ (red). The calculated center of masses for each modulation, shown as a black solid line, correspond to the arrival times of 500 individual bunches with respect to the laser pulses. One can clearly see a shift of the arrival time of about $8 \mathrm{ps}$ for the 500 consecutive bunches. The color code also indicates a change of the bunch lengths (rms) from $10 \mathrm{ps}$ to $6 \mathrm{ps}$.

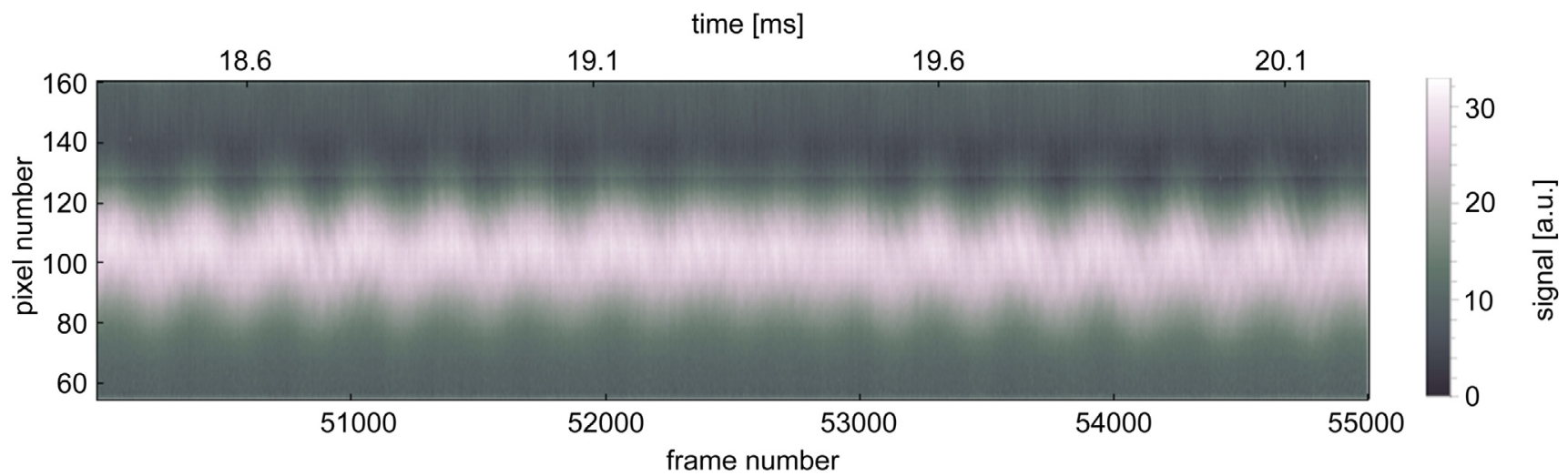

Fig. 3. Raw measurement data of the longitudinal bunch profile recorded with KALYPSO at the EOSD at KARA. The color code corresponds to the relative modulation of the laser spectrum. Only $5 \cdot 10^{3}$ frames are plotted to highlight the effect of micro-bunching instabilities on the longitudinal bunch profile. The full dataset consists of nearly $10^{7}$ frames. 


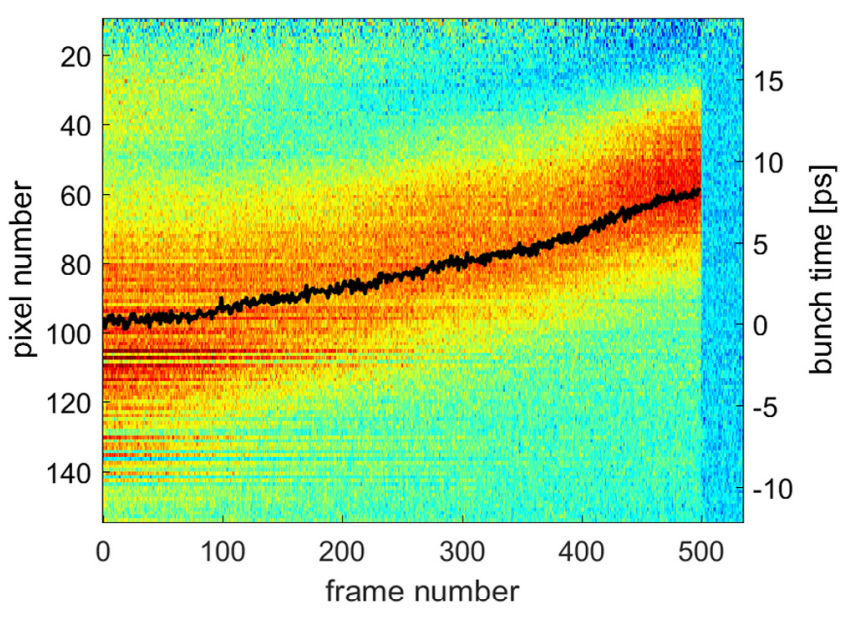

Fig. 4. 526 consecutive frames recorded with a rate of $1.13 \mathrm{MHz}$. The frames show the modulation of the spectrum of a pulsed laser synchronized to the electron bunches at the Eu-XFEL. The color code corresponds to a modulation depth from $-5 \%$ (blue) to $+10 \%$ (red). The center of mass of the modulation is shown as a black line, which indicates the arrival times of 500 individual bunches. (For interpretation of the references to color in this figure legend, the reader is referred to the web version of this article.)

\section{Conclusion and outlook}

We have developed KALYPSO, a line scan detector for visible light and near-infrared radiation operating with a frame-rate of up to $2.7 \mathrm{MHz}$. To the best of the author's knowledge, this is the highest frame rate achieved by high-resolution imaging detectors with continuous data acquisition. The detector has been installed at the EOSD experimental setups at KARA and at the European XFEL. Several technical developments have been started to further improve the performance of the detector system. Dedicated Si micro-strip sensors with different antireflective coatings and an optimized geometry are being integrated with the KALYPSO detector. Moreover, to further increase the frame rate and improve the noise performance of KALYPSO, a dedicated readout ASIC is being developed. The results of the first prototype of the new ASIC with 48 channels have been reported in [15].

\section{Acknowledgments}

This project is partially funded by the BMBF, Germany contract number 05K16VKA. The authors would like to thank Simon Kudella, Fabio Colombo and Pia Steck for their help with the wire-bonding process.

\section{References}

[1] A. Borysenko, et al., Electron bunch shape measurements using electro-optical spectral decoding, Phys. Proc. 77 (2015) 3-8.

[2] B. Steffen, et al., Electro-optic time profile monitors for femtosecond electron bunches at the soft x-ray free-electron laser FLASH, Phys. Rev. Spec. Top. Accel. Beams (ISSN: 1098-4402) 12 (3) (2009) http://dx.doi.org/10.1103/PhysRevSTAB. 12.032802 .

[3] B. Henrich, et al., The adaptive gain integrating pixel detector AGIPD a detector for the European XFEL, Nucl. Instrum. Methods Phys. Res. A (ISSN: 0168-9002) 633 (2011) S11 - S14, http://dx.doi.org/10.1016/j.nima.2010.06.107.

[4] A Mozzanica, et al., The GOTTHARD charge integrating readout detector: design and characterization, J. Instrum. 7 (01) (2012) C01019.

[5] M. Caselle, et al., A high-speed daq framework for future high-level trigger and event building clusters, J. Instrum. 12 (03) (2017) C03015.

[6] L. Rota, et al., A high-throughput readout architecture based on PCI-Express Gen3 and DirectGMA technology, J. Instrum. 11 (02) (2016) P02007.

[7] L. Rota, et al., A PCIe DMA architecture for multi-gigabyte per second data transmission, IEEE Trans. Nucl. Sci. 62 (3) (2015) 972-976.

[8] M. Vogelgesang, et al., A heterogeneous FPGA/GPU architecture for real-time data analysis and fast feedback systems, in: 5th Int. Beam Instrumentation Conf.(IBIC'16), Barcelona, Spain, Sept. 13-18, 2016, 2017, pp. 627-630.

[9] P Schönfeldt, et al., Towards near-field electro-optical bunch profile monitoring in a multi-bunch environment, in: Proceedings of IPAC2017, Copenhagen, Denmark, MOPAB055, 2017, pp. 227-230.

[10] G. Niehues, et al., High repetition rate, single-shot electro-optical monitoring of longitudinal electron bunch dynamics using the linear array detector KALYPSO, in: 9th Int. Particle Accelerator Conf. (IPAC'18), Vancouver, BC, Canada, 2018, p. WEPAL026.

[11] B. Steffen, V. Schlott, F. Müller, A compact single shot electro-optical bunch length monitor for the SwissFEL, in: Proceedings of DIPAC09, Basel, Switzerland, 2009.

[12] A. Mielczarek, et al., Real-time data acquisition and processing system for $\mathrm{MHz}$ repetition rate image sensors, IEEE Trans. Nucl. Sci. (ISSN: 0018-9499) in print (2018).

[13] U. Mavrič, et al., Precision synchronization of optical lasers based on MTCA.4 electronics, in: Proceedings of IBIC2013, 2013, Oxford, UK.

[14] M. Felber, et al., Compact MTCA.4 based laser synchronization, in: Proceedings of IPAC2014, Dresden, Germany, 2014.

[15] L. Rota, et al., Development of a front-end ASIC for 1D detectors with $12 \mathrm{mhz}$ frame-rate, in: Proceedings, Topical Workshop on Electronics for Particle Physics (TWEPP17): Santa Cruz, CA, USA, September 11-15, 2017, PoS TWEPP-17 (2017) 033, http://dx.doi.org/10.22323/1.313.0033. 